\title{
A Criterion for Hurwitz Polynomials and its Applications
}

\author{
Liejun Xie \\ Department of Mathematics, Ningbo University, Zhejiang Ningbo 315211, China \\ Email: xieliejun@nbu.edu.cn
}

\begin{abstract}
We present a new criterion to determine the stability of polynomial with real coefficients. Combing with the existing results of the real and negative roots discrimination, we deduced the explicit conditions of stability for any real polynomial with a degree no more than four. Meanwhile, we discussed the problem of controls system stability and inertia of Bezout matrix as the applications of the criterion. A necessary and sufficient condition to determine the stability of the characteristic polynomial of the continuous time control systems was proposed. And also, we discussed a pathological case of the bilinear transformation, which can convert the stability analysis of a given discr ete time system to the corresponding continuous time system, and brought forward an alter native one.
\end{abstract}

Index Terms-Hurwitz polynomial, stability, control system, inertia of Bezout matrix.

\section{INTRODUCTION}

$$
\text { Let } f(\omega)=a_{0} \omega^{n}+a_{1} \omega^{n-1}+\ldots+a_{n-1} \omega+a_{n}
$$

is a polynomial of degree $n$ with real coefficients, then it is said to be a stable or a Hurwitz polynomial if and only all its roots lie in the open left half of the complex plane. i.e. $f(\omega)=0 \Rightarrow \operatorname{Re} \omega<0$.

The well-known Routh-Hurwitz Theorem provides a powerful tool to check if a real polynomial is stable or not.

The Routh-Hurwitz Criterion. (See [1].) The polynomial (1) is stable if, and only if, the following inequalities holds: $\Delta_{1}>0, \Delta_{2}>0, \ldots, \Delta_{n}>0$, where

$$
\Delta_{k}=\left|\begin{array}{cccccccc}
a_{1} & a_{0} & 0 & 0 & 0 & 0 & \ldots & 0 \\
a_{3} & a_{2} & a_{1} & a_{0} & 0 & 0 & \ldots & 0 \\
a_{5} & a_{4} & a_{3} & a_{2} & a_{1} & a_{0} & \ldots & 0 \\
a_{7} & a_{6} & a_{5} & a_{4} & a_{3} & a_{2} & \ldots & 0 \\
M & M & M & M & M & M & O & M \\
a_{2 k-1} & a_{2 k-2} & a_{2 k-3} & a_{2 k-4} & a_{2 k-5} & a_{2 k-6} & \ldots & a_{k}
\end{array}\right| .
$$

Supported by the National Science Foundation of Ningbo City (No.2009A610078), and the research foundation of Ningbo University (No.xk109042, xk109043). usually, $\Delta_{\mathrm{k}}(\mathrm{k}=1,2, \ldots, \mathrm{n})$ named as the Hurwitz determinant. Whereas the inequalities $\Delta_{k}>0(k=1,2, \ldots, n)$ can be simplified when all of the coefficients of polynomial (1) are positive. The relative result was deduced by Li nard and Chipart in 1914.

The Li enard-Chipart Criterion. (See [2].)Consider a real polynomial $f(x)=x^{n}+C_{1} x^{n-1}+C_{2} x^{n-2}+\ldots+C_{n}$, it is stable if and only if the one of following cases holds:

(1) $C_{n}>0, C_{n-2}>0, \ldots ; \Delta_{1}>0, \Delta_{3}>0, \ldots$

(2) $C_{n}>0, C_{n-2}>0, \ldots ; \Delta_{2}>0, \Delta_{4}>0, \ldots$

(3) $\mathrm{C}_{\mathrm{n}}>0, \mathrm{C}_{\mathrm{n}-1}>0, \ldots ; \Delta_{1}>0, \Delta_{3}>0, \ldots$

(4) $C_{n}>0, C_{n-1}>0, \ldots ; \Delta_{2}>0, \Delta_{4}>0, \ldots$

Polynomial stability problems of various types arise in a number of problems in mathematics and engineering. We refer to [1, Chapter 15] for deep surveys on the classical stability theory and $[3,4,5,6,7]$ of recent results.

In this paper, we present a new criterion for determining the Hurwitz polynomial.

The rest of this paper is organized in the following ways with the next section stating the main result. And also, we present some computation examples; deduce the explicit criterion for stability of real polynomials with degree no more than four, which is expressed by polynomials in the coefficient of the given polynomial.

In section III, two problems are discussed as the application of our criterion. The first problem is relative to the stability of the control system. In classical theory of control systems stability analysis, determining the stability of a given discrete time system can be converted to the stability of the corresponding continuous time system through a bilinear transformation. We introduced a necessary and sufficient condition to determine the stability of the characteristic polynomial of the continuous time control systems in subsection A. In subsection B, we discussed a pathological case of the bilinear transformation used widely, and brought forward an alternative one. The second problem, which is relative to the inertia of Bezout matrix, was discussed in subsection C. An algorithm named as INER was brought forward.

\section{A CRITERION FOR HURWITZ POLYNOMIALS}




\section{A. Criterrion}

Without loss of generality, supposed that the real polynomial $f(\omega)=\omega^{n}+a_{1} \omega^{n-1}+\ldots+a_{n}$ is monic. We construct $f(\omega)=f(x+i y)=f_{1}(x, y)+i \cdot f_{2}(x, y)$, and get two real polynomials $f_{1}(x, y), f_{2}(x, y)$.

Let $f_{1}(x, y)$ and $f_{2}(x, y)$ be expressed respectively as follows:

$$
\begin{aligned}
& \mathrm{f}_{1}(\mathrm{x}, \mathrm{y})=\alpha_{0} \mathrm{y}^{\mathrm{k}}+\alpha_{1} \mathrm{y}^{\mathrm{k}-1}+\ldots+\alpha_{\mathrm{k}}, \\
& \mathrm{f}_{2}(\mathrm{x}, \mathrm{y})=\beta_{0} \mathrm{y}^{\mathrm{m}}+\beta_{1} \mathrm{y}^{\mathrm{m}-1}+\ldots+\beta_{\mathrm{m}},
\end{aligned}
$$

Where $\mathrm{k}, \mathrm{m} \leq \mathrm{n}, \mathrm{k}+\mathrm{m}=2 \mathrm{n}-1$ and $\alpha_{\mathrm{i}}, \beta_{\mathrm{j}}$ are all the real polynomial with variable $X$. It is not difficult to verify the changes of $\alpha_{0}, \beta_{0}$ according to $n$.

Table 1 the changes of $\alpha_{0}, \beta_{0}$ according to $\mathrm{n}$

\begin{tabular}{lllll}
\hline $\mathrm{n}$ & 2 & 3 & 4 & 5 \\
\hline$\alpha_{0}$ & -1 & $-\mathrm{a}_{1}-3 \mathrm{x}$ & 1 & $\mathrm{a}_{1}+5 \mathrm{x}$ \\
$\beta_{0}$ & $\mathrm{a}_{1}+2 \mathrm{x}$ & -1 & $-\mathrm{a}_{1}-4 \mathrm{x}$ & 1 \\
\hline $\mathrm{n}$ & 6 & 7 & 8 & $\ldots$ \\
\hline$\alpha_{0}$ & 1 & $-\mathrm{a}_{1}-7 \mathrm{x}$ & 1 & $\cdots$ \\
$\beta_{0}$ & $\mathrm{a}_{1}+6 \mathrm{x}$ & -1 & $-\mathrm{a}_{1}-8 \mathrm{x}$ & $\ldots$ \\
\hline
\end{tabular}

Then we compute the resultant of $f_{1}(x, y)$ and $f_{2}(x, y)$ with respect to $y$, denoted by $\operatorname{res}\left(f_{1}, f_{2}, y\right)$ marked (5).

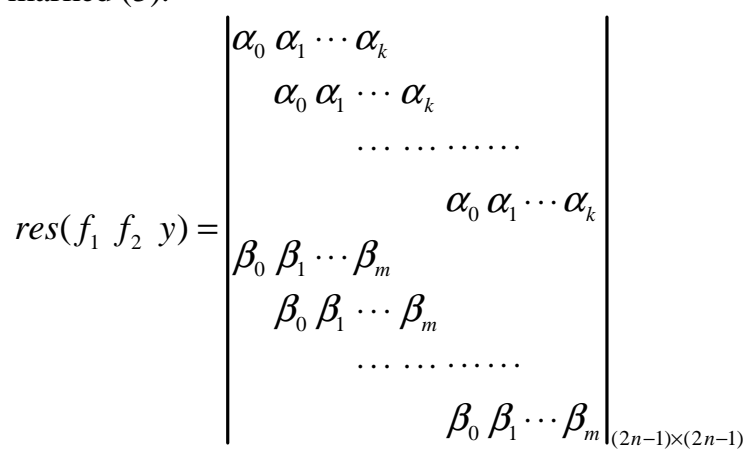

The following lemma gives the relation between the real roots of $\operatorname{res}\left(f_{1}, f_{2}, y\right)$ and the $x$-coordinates of the solutions of PS (Polynomials System):

$$
\left\{f_{1}(x, y)=0, f_{2}(x, y)=0\right\}
$$

Lemma 1. (See [8].) In general, $r e s\left(f_{1}, f_{2}, y\right)$ is a polynomial in $X$ whose roots are the $X$ - coordinates of the solutions of PS. According to the classical result from Algebra, it is well known:

Lemma 2. (See [9].) $\operatorname{res}\left(f_{1}, f_{2}, y\right)=0$ if and only if $f_{1}(x, y)$ and $f_{2}(x, y)$ have a common factor in ${ }^{\circ}[x, y]$, or $\alpha_{0}=\beta_{0}=0$.

Let $I C\left(f_{1}, y\right)$ and $I C\left(f_{2}, y\right)$, which are all the polynomials with real coefficients in $x$, be the leading coefficient of the polynomial $f_{1}(x, y)$ and $f_{2}(x, y)$ in $y$. And also we denote the greatest common factor of
IC $\left(f_{1}, y\right)$ and IC $\left(f_{2}, y\right)$ by $G L C_{x}\left(f_{1}, f_{2}\right)$. Obviously, $G L C_{x}\left(f_{1}, f_{2}\right)$ is a polynomial in $x$, too.

Theorem 1. Denote the number of distinct real roots of $\operatorname{res}\left(\mathrm{f}_{1}, \mathrm{f}_{2}, \mathrm{y}\right)$ and $\mathrm{GLC}_{\mathrm{x}}\left(\mathrm{f}_{1}, \mathrm{f}_{2}\right)$ by $\mu$ and $\mathrm{m}$, respectively, and the numbers of distinct $x$ - coordinates of the solutions of PS by $\gamma$, then $\gamma=\mu-\mathrm{m}$. Especially, if $\mathrm{GLC}_{\mathrm{x}}\left(\mathrm{f}_{1}, \mathrm{f}_{2}\right)=1$, then we have $\gamma=\mu$.

Proof. It is obvious according to Lemma 1 and Lemma 2.

Denote the set of distinct $x$ - coordinates of the solutions of PS by $\operatorname{Zer}_{x}(P S)$, the distinct roots of $\operatorname{res}\left(f_{1}, f_{2}, y\right)$ by Zero $\left(r e s\left(f_{1}, f_{2}, y\right)\right)$, and the distinct roots of $G L C_{x}\left(f_{1}, f_{2}\right)$ by Zero $\left(G L C_{x}\left(f_{1}, f_{2}\right)\right)$.

In general, $\operatorname{Zer}_{x}(P S) \subseteq \operatorname{Zero}\left(\operatorname{res}\left(f_{1}, f_{2}, y\right)\right)$.From Theorem 1 it is easy to see that:

Corollary 1. Zero ${ }_{x}(P S) \cup \operatorname{Zero}\left(G_{L} C_{x}\left(f_{1}, f_{2}\right)\right)=$ Zero(res $\left.\left(f_{1}, f_{2}, y\right)\right)$.

Denote the distinct real roots of res $\left(f_{1}, f_{2}, y\right)$ and the distinct negative real roots of $\operatorname{res}\left(f_{1}, f_{2}, y\right)$ by $I$ and $m$, respectively. Then we deduce the main result in following: Theorem 2. $f(\omega) \in H[f]$ if and only if $I=m \neq 0$.

Proof. Firstly, $I=m \neq 0$ implies all the real roots of $\operatorname{res}\left(f_{1}, f_{2}, y\right)$ are negative. Secondly, according to table $1, G L C_{x}\left(f_{1}, f_{2}\right)=1$. Combining corollary 1 , we deduce that $\operatorname{Zer}_{x}(P S)=\operatorname{Zero}\left(\operatorname{res}\left(f_{1}, f_{2}, y\right)\right)$. Thus, all of the roots of polynomial $f(\omega)$ have negative real part. That is to say $f(\omega) \in H[f]$.

B. Explicit condition of stability for any real polynomial with degree no more than four

In this subsection, we will deduce the explicit condition of stability for any real polynomial with a degree no more than four. According to Theorem 2, it is important to analyze the number of distinct real roots and distinct negative real roots of a real polynomial. Let's introduce the relative results from [10], [11] and [12].

Definition 1. Given a polynomial with real coefficients

$$
f(x)=\sum_{i=0}^{n} a_{i} x^{n-i}\left(a_{0} \neq 0\right),
$$

The following $(2 n+1) \times(2 n+1)$ matrix is called the discrimination matrix of and denoted by $\operatorname{Discr}(f)$. 


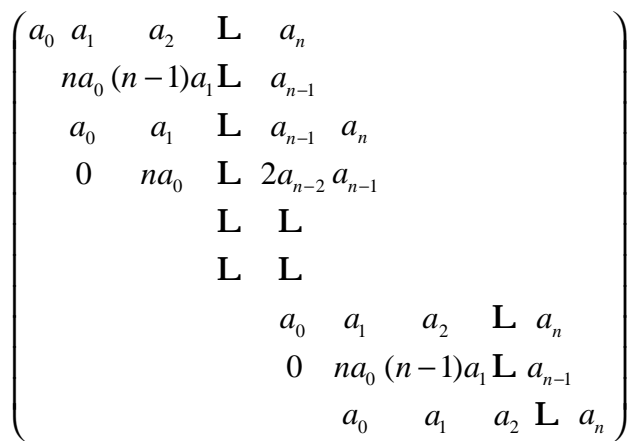

Definition 2. Let $D_{k}=d_{2 k}$ for $k=1,2, \ldots n$, We called $n-$ tuple $\left\{D_{1}(f), D_{2}(f), \ldots D_{n}(f)\right\}$ the discrimination sequence of polynomial $f(x)$.

Definition 3. By $\left\{d_{1}, d_{2}, \ldots, d_{2 n+1}\right\}$ denote the principal minor sequence of Discr ( $f)$, we call the $2 n$-tuple $\left\{d_{1} d_{2}, d_{2} d_{3}, \ldots, d_{2 n} d_{2 n+1}\right\}$ the negative root discrimination sequence, and denote it by n.r.d $(f)$.

Definition 4. We call $\left\{\operatorname{sign}\left(D_{1}\right), \operatorname{sign}\left(D_{2}\right), \ldots\right.$, $\left.\operatorname{sign}\left(D_{n}\right)\right\}$ the sign list of a given sequence $\left\{D_{1}(f)\right.$, $\left.D_{2}(f), \ldots D_{n}(f)\right\}$, where $\operatorname{sign}(\cdot)$ is a sign function.

Definition 5. Given a sign list $\left[\mathrm{s}_{1}, \mathrm{~s}_{2}, \ldots, \mathrm{s}_{\mathrm{n}}\right]$, we construct its revised sign list, $\left[\mathrm{t}_{1}, \mathrm{t}_{2}, \ldots, \mathrm{t}_{\mathrm{n}}\right]$, as follows:

1) if $\left[S_{i}, S_{i+1}, \ldots, S_{i+j}\right]$ is a section of given list, where $\mathrm{S}_{i} \neq 0, \mathrm{~S}_{i+1}=\cdots=\mathrm{S}_{i+j-1}=0, \mathrm{~S}_{i+j} \neq 0$, then we replace the subsection $\left[\mathrm{S}_{\mathrm{i}+1}, \mathrm{~S}_{\mathrm{i}+2}, \cdots, \mathrm{S}_{\mathrm{i}+j-1}\right]$ by the first terms of $\left[-s_{i},-s_{i}, s_{i}, s_{i},-s_{i},-s_{i}, s_{i}, s_{i}, \cdots\right]$.

2) Otherwise, let $t_{k}=s_{k}$, i.e. no changes for the others.

Lemma 3. Given a polynomial $f(x)$ with real coefficients, if the numbers of the sign changes of the revised sign of $\left\{D_{1}(f), D_{2}(f), \ldots D_{n}(f)\right\}$ is $\mu$, then the number of the pairs of distinct conjugate imaginary roots of $\mathrm{f}(\mathrm{x})$ equals to $\mu$. Furthermore, if the number of nonvanishing members of revised sign list is $I$, then, the number of the distinct real root of $f(x)$ equals $\mid-2 \mu$.

Lemma 4. Let $\left\{d_{1}, d_{2}, \ldots, d_{2 n+1}\right\}$ be the principal minor sequence of $\operatorname{Discr}(f)$, the discrimination matrix of polynomial $f(x)$ with $f(0) \neq 0$. Denote the number of sign changes and the number of non-vanishing members of revised sign of sequence $\left\{d_{1} d_{2}, d_{2} d_{3}, \ldots, d_{2 n} d_{2 n+1}\right\}$ by $\mu$ and $2 \mathrm{~m}$, respectively. Then, the number of distinct negative roots of $f(x)$ equals to $\mu-2 m$.

According to the Lemma 3 and Lemma 4, we can give the explicit condition to describe the number of the distinct real roots and the negative roots.
Let us consider the following polynomial with real coefficients. All the computations are made with the Computer Algebraic System MAPLE14.

$$
\begin{aligned}
& \mathrm{f}_{1}(\omega)=\omega^{2}+a_{1} \omega+a_{2} \\
& f_{2}(\omega)=\omega^{3}+a_{1} \omega^{2}+a_{2} \omega+a_{3} \\
& f_{3}(\omega)=\omega^{4}+a_{1} \omega^{3}+a_{2} \omega^{2}+a_{3} \omega+a_{4}
\end{aligned}
$$

Using the method discussed above, it is not difficult to establish the explicit criterion for stability, which is expressed by polynomials in the coefficient of the given polynomial.

1) $f_{1}(\omega) \in H[f]$ if and only if $a_{1}>0, a_{2}>0$.

2) $f_{2}(\omega) \in H[f]$ if and only if $a_{1}>0, a_{1} a_{2}>a_{3}, a_{3}>0$.

3) $f_{3}(\omega) \in H[f]$ if and only if $a_{1}>0, a_{1} a_{3}+a_{2}^{2}-4 a_{4}$ $>0, a_{1} a_{2} a_{3}-a_{1}^{2} a_{4}-a_{3}^{2}>0, a_{4}>0$.

\section{APPLICATIONS}

\section{A. The stability of control system}

The stability analysis is one of the most important aspects in the design of control systems. A discrete time system is called stable if all the zeros of the system characteristic polynomial lie in the unit circle. There are some methods to check the stability of a given discrete time system [13][14]. However, in some cases, instead of direct analysis on the discrete time system, it is more convenient to perform stability analysis on an equivalent continuous time system. The stability of a continuous time system is determined by the root locations of the system characteristic polynomial with respect to the imaginary axis, the system being stable if and only if all roots lie in the open left half complex plane. The characteristic polynomial of a discrete time system is called Schur stability if the corresponding discrete system is stable, and the same as Hurwitz stability of a continuous system characteristic polynomial. There exist also many methods to check the stability of a given continuous time system [1][2][15]. Using a bilinear transformation, the determination of Schur stability can be converted to the determination of Hurwitz stability of an equivalent polynomial. As a matter of convenience, we marked $f(z) \in S[f]$ if $f(z)$ being Schur stability, and $g(w) \in H[g]$ if $g(\omega)$ being Hurwitz stability.

Now we can describe our algorithm for determining the Hurwitz stability of the characteristic polynomial of a continuous time system as follows. The correctness of the algorithm is guaranteed by the above discussion. Without loss of generality, we assume that the polynomials in following algorithm have no multiplicative roots. Otherwise, we may use the algorithm [11][16] to determine the multiplicities of real roots for a given polynomial.

Step 1. Input the monic polynomial $f(\omega)$, and then obtain the polynomial $f_{1}(x, y)$ and $f_{2}(x, y)$ by acting the substitution $\omega=x+i \cdot y$ upon $f(\omega)$. 
Step 2. Compute the resultantres $\left(f_{1}, f_{2}, y\right)$, and then analysis the distinct real roots of $\operatorname{res}\left(f_{1}, f_{2}, y\right)$, which denoted byl, by using Lemma 3. Analysis the distinct negative real roots of $r e s\left(f_{1}, f_{2}, y\right)$, which denoted by $m$, by using Lemma 4.

Step 3. Deduce the conclusion by the Theorem 2 .

Our method has been implemented by using Computer Algebraic System MAPLE14 for numerical and algebraic manipulations. The following example illustrated our approach.

Example 1. Consider the determination of Hurwitz stability of the following continuous time polynomial

$$
f(\omega)=\omega^{3}-\omega+1 \text {. }
$$

It is not difficult to deduce that $I=2$ and $m=1$. Thus, according to Theorem 2, the polynomial $f(\omega)$ is not Hurwitz stability. That is to say, the corresponding continuous time system is not stable.

Example 2. Consider the determination of Hurwitz stability of the following polynomial

$f(\omega)=2.16 \omega^{4}-0.42 \omega^{3}+6.58 \omega^{2}-0.42 \omega+2.16$.

$\mathrm{I}=2$ and $\mathrm{m}=0$. According to Theorem 2, $\mathrm{f}(\omega)$ is not Hurwitz stable.

Example 3. Consider the determination of Hurwitz stability of the following polynomial

$f(\omega)=1.70396616 \omega^{8}+4.49238296 \omega^{7}+12.29115880$.

$$
\begin{aligned}
& \omega^{6}+16.93754704 \omega^{5}+22.04234384 \omega^{4}+ \\
& \text { 16.93754704 } \omega^{3}+12.29115880 \omega^{2}+ \\
& 4.49238296 \omega+1.70396616 .
\end{aligned}
$$

$I=m=2$. According to Theorem 2, $f(\omega)$ is Hurwitz stable.

\section{B. The pathological case of the bilinear transformation}

Denote

$$
g(\omega)=f\left(\frac{\omega+1}{\omega-1}\right)(\omega-1)^{n},
$$

Then $f(z) \in S[f]$ if and only if $g(w) \in H[g]$.

However, there exists a pathological case while using bilinear transformation to determine the Schur stability via the Hurwitz stability.

Considering the following discrete-time polynomial:

Example 4. $f(z)=z^{3}-1.21 z^{2}-0.20 z+0.41$

Using the bilinear transformation

$$
z=(\omega+1) /(\omega-1)
$$

We obtain

$$
g(w)=f\left(\frac{w+1}{w-1}\right)(w-1)^{3}=0.76 w^{2}+5.64 w+1.6 .
$$

It has two real roots

$$
\mathrm{w}_{1}=-0.2954505652, \mathrm{w}_{2}=-7.125602066 \text {. }
$$

Thus, $g(w) \in H[g]$, furthermore, $f(z) \in S[f]$. However, the above polynomial $f(z)$ has a root at $z=1$, which leads to non-Schur stability. Indeed, there exist much more examples verifying the pathological case while the discrete time polynomial has a root at $Z=1$.

Let us introduce an alternative transformation without any question.

Given a polynomial with real coefficients

$$
f(z)=\sum_{i=0}^{n} a_{i} z^{n-i}\left(i=0,1,2, \ldots, n ; a_{0} \neq 0\right) .
$$

Using the transformation

$$
z=\left(1+\omega+\omega^{2}\right) /\left(1-\omega+\omega^{2}\right),
$$

Then we get the polynomial

Where

$$
g_{0}(\omega)=g(\omega) /\left(1-\omega+\omega^{2}\right)^{n},
$$

$g(\omega)=b_{0} \omega^{2 m}+b_{1} \omega^{2 m-1}+\ldots+b_{2 m}\left(b_{j} \in^{\circ}, j=0,1,2, \ldots, 2 m\right)$ and $m \leq n$.

Let $\omega=x+i y$, which leads to

$$
|z|^{2}=\frac{\left|1+\omega+\omega^{2}\right|^{2}}{\left|1-\omega+\omega^{2}\right|^{2}}=\frac{\left(1+x+x^{2}-y^{2}\right)^{2}+(2 x y+y)^{2}}{\left(1-x+x^{2}-y^{2}\right)^{2}+(2 x y-y)^{2}} .
$$

Denote $g(x, y)$ as the expression when the numerator minus the denominator of (18), it is easy to simplify that

$$
g(x, y)=4 x\left(x^{2}+y^{2}+1\right)
$$

Combining (18) and (19), we obtain the following result

1) $|z|<1 \dot{E} \quad g(x, y)<0 \dot{E} \quad x<0 \dot{E} \quad \omega$ lies in the open left half plane;

2) $|z|=1 \dot{E} \quad g(x, y)=0 \dot{E} \quad x=0 \hat{E} \quad \omega$ lies in the imaginary axis;

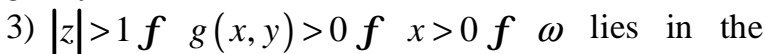
open right half plane.

According to the discussion above, we obtain the following theorem.

Theorem 3. The transformation (16) maps the imaginary axis to the unit circle and vice versa, the open left half plane to the inside of unit circle and vice versa, the open right half plane to the outside of unit circle and vice versa.

Theorem 4. $f(z) \in S[f] E ́ \quad g(\omega) \in H[g]$, where the definitions of $f(z)$ and $g(\omega)$ are the same as mentioned above.

According to Theorem 4, determining the stability of a given discrete time system can be converted to the stability of the corresponding continuous time system through the transformation (16).

Now, we reconsider example 4. By using (16), we obtain

$$
g(\omega)=0.76 \omega^{5}+5.64 \omega^{4}+3.12 \omega^{3}+5.64 \omega^{2}+0.76 \omega
$$

Obviously, $g(0)=0$, thus $\mathrm{g}(\omega) \notin \mathrm{H}[\mathrm{g}]$. According to Theorem 4, f $(\mathrm{z}) \notin \mathrm{S}[\mathrm{f}]$.

Example 5. Consider the determination of Schur stability of the following discrete time polynomial

$$
f(z)=z^{2}-0.05 z+1.21 \text {. }
$$


Using the transformation (16), we have

$$
\begin{aligned}
g(\omega)= & 2.16 \omega^{4}-0.42 \omega^{3}+6.58 \omega^{2}- \\
& 0.42 \omega+2.16 \notin \mathrm{H}[\mathrm{g}] .
\end{aligned}
$$

Thus, $f(z) \notin S[f]$.

Example 6. Consider the determination of Schur stability of the following discrete time polynomial

$$
f(z)=z^{4}+0.338 z^{3}+0.28006 z^{2}+0.0800038 z+
$$

$$
0.00590236
$$

Using the transformation (16), we have

$$
\begin{aligned}
g(\omega)= & 1.70396616 \omega^{8}+4.49238296 \omega^{7}+ \\
& 12.29115880 \omega^{6}+16.93754704 \omega^{5}+ \\
& 22.04234384 \omega^{4}+16.93754704 \omega^{3}+ \\
& 12.29115880 \omega^{2}+4.49238296 \omega+ \\
& 1.70396616
\end{aligned}
$$

The above polynomial has four pairs of conjugate imaginary roots which are all own negative imaginary part. As a result, the corresponding continuous time system is stable. Thus, the discrete time system considered is stable, too.

\section{The inertie of Bezout matrix}

Let $u(x)$ and $v(x)$ be two polynomial in integer domain of degree $n$ and $m, n \geq m$, respectively,

$$
u(x)=\sum_{i=0}^{n} u_{i} x^{i}, v(x)=\sum_{i=0}^{m} v_{i} x^{i}, u_{n}, v_{m}(x) \neq 0 .
$$

A $n \times n$ Bezout matrix $B=\left(b_{i, j}\right) \in \Phi^{n \times n}$ associated with $u(x)$ and $v(x)$ is defined by

$$
\frac{u(x) v(y)-u(y) v(x)}{x-y}=\sum_{i, j=1}^{n} b_{i, j} x^{i-1} y^{j-1} .
$$

The entries of $B$ can recursively be computed by means of the formula

$$
b_{i, j+1}=b_{i+1, j}+u_{i} v_{j}-u_{j} v_{i}
$$

Complemented with the initial conditions $b_{i, 0}=b_{n+1, j}=$ 0 . This rule clearly shows that the entries of $B$ are polynomials of degree 2 in the coefficients of $u(x)$ and $v(x)$.

Definition 6. (See [17][18].) The inertia of Bezout matrix $B$ is defined by a triple

$$
\ln (B)=(\pi(B), v(B), \delta(B)),
$$

where $\pi(\mathrm{B}), \mathrm{v}(\mathrm{B})$ and $\delta(\mathrm{B})$ are, respectively, the numbers of Eigen values of $B$ with positive, negative, and zero real parts.

It is well known that all the classical tools for investigation of the roots of algebraic equations, such as the Sturm theorem and continued-fraction criteria, can be proved purely algebraically by computing the inertia of Hankel and Bezout matrices associated with suitable real polynomial $u(x)$ and $v(x)$ of degree $n$ and less than $n$, respectively[19][20]. In [20], the authors solved certain classical zero-location problems with the help of the inertia of Bezout and Hankel matrix.
In [18], the author revealed the relation between the inertia of Bezout matrix associated with $u(x)$ and $v(x)$ and the signs of leading coefficients of every quotient in quotient sequence generated by using Euclidean scheme to real polynomials $u(x)$ and $v(x)$.

In [21] the evaluation of the inertia of real Bezout matrix is obtained by computing a block LDL $t$ factorization where $\mathrm{L}$ is a lower triangular matrix with unit diagonal entries and $\mathrm{D}$ is a block diagonal matrix. By Sylvester's law[19], the factorization preserves the inertia, moreover, the diagonal blocks in $\mathrm{D}$ are themselves Hankel matrices, so their inertia can be computed by direct inspection using a set of rules given by Iochvidov's rule[22]. However, it is difficult to control the growth of coefficient $s$ in these algorithms.

A fast and fraction-free procedure for computing the inertia of Bezout matrix was presented in [23], and also it derived the new method to determining the numbers of different real roots and different pairs of conjugate complex roots of a polynomial with integer coefficients. Yet it depends on a fast method to determine the signs of the leading coefficients of every quotient in quotient sequence generated by applying Euclid's algorithm.

In this section, based on the discussion and results in section II, we proposed a new method to compute the inertia of Bezout matrix with polynomials $u(x)$ and $\mathrm{v}(\mathrm{x})$.

\section{Algorithm: INER}

Input: two polynomials $u(x)$ and $v(x)$ with integer coefficients.

Output: the inertia of Bezout matrix associated with $u(x)$ and $(x)$.

Step 1. Compute the Bezout matrix $B$ associated with $u(x)$ and $v(x)$ according to the formula (24), then get characteristic polynomial $f(\omega)$ of it. Using the transformation $\omega=x+i y$ upon $f(\omega)$, we can get two bivarate polynomials $f_{1}(x, y)$ and $f_{2}(x, y)$ denoted by (3) and (4), respectively. And also, we can deduce the $I c\left(f_{1}, y\right), I C\left(f_{2}, y\right)$ and $\operatorname{res}\left(f_{1}, f_{2}, y\right)$ mentioned above.

Step 2. Analysis the numbers of zero solution of $\operatorname{GLC} C_{x}\left(f_{1}, f_{2}\right)$ andres $\left(f_{1}, f_{2}, y\right)$, denoted by $n_{1,0}$ and $n_{2,0}$, respectively.

Step 3. Let

$$
G L C_{f_{1}, f_{2}}(x)=\frac{G L C_{x}\left(f_{1}, f_{2}\right)}{\operatorname{gcd}\left(G L C_{x}\left(f_{1}, f_{2}\right), \operatorname{diff}\left(G L C_{x}\left(f_{1}, f_{2}\right), x\right)\right)}
$$

Compute the number of distinct negative roots, denoted by $n_{1,-}$, by using Lemma 4 to $G L C_{x}\left(f_{1}, f_{2}\right)$. Applying Lemma 3, we can obtain the number of distinct roots of $\operatorname{GLC}_{x}\left(f_{1}, f_{2}\right)$, denoted by $\mu$. Then, the number of positive roots is $\mu-n_{1,-}$, denoted by $n_{1,-}$.

Step 4. Let 


$$
\operatorname{res}_{f_{1}, f_{2}}(x)=\frac{\operatorname{res}\left(f_{1}, f_{2}, y\right)}{\operatorname{gcd}\left(\operatorname{res}\left(f_{1}, f_{2}, y\right)\right), \operatorname{diff}\left(\operatorname{res}\left(f_{1}, f_{2}, y\right), x\right)}
$$

Then, $n_{2,+}$ and $n_{2,-}$ will be obtained, when we apply the same method of Step 3 to the polynomial $\operatorname{res}_{\mathrm{f}_{1}, \mathrm{f}_{2}}(\mathrm{x})$.

Step 5. Compute the inertia

$$
\left(n_{2,+}-n_{1,+}, n_{2,0}-n_{1,0}, n_{2,-}-n_{1,-}\right) \text {. }
$$

Our method has been implemented by using the routines by MAPLE14 for numerical and algebraic manipulations. The following two examples illustrate our approach.

Example 4. (See [24].) Let us consider the $4 \times 4$ Bezout matrix $B$ associated with the polynomials

$$
u(x)=3+4 x^{3}+8 x^{4}
$$

and

$$
v(x)=1+2 x+4 x^{2}
$$

We find that the Bezout matrix associated with $u(x)$ and $v(x)$ is

$$
\left[\begin{array}{cccc}
-6 & -12 & 4 & 8 \\
-12 & 4 & 16 & 16 \\
4 & 16 & 32 & 32 \\
8 & 16 & 32 & 0
\end{array}\right]
$$

And the characteristic polynomials is

$$
f(\omega)=\omega^{4}-30 \omega^{3}-1848 \omega^{2}-3008 \omega+176128 \text {. }
$$

Using transformation $\omega=\mathrm{x}+\mathrm{iy}$, we get the following polynomials:

$$
\begin{aligned}
f_{1}(x, y)=y^{4}+ & \left(-6 x^{2}+90 x+1848\right) y^{2}+ \\
& x^{4}-30 x^{3}+1848 x^{2}-3008 x+ \\
& 176128
\end{aligned}
$$

and

$$
\begin{aligned}
f_{2}(x, y)= & (-40 x+30) y^{3}+ \\
& \left(4 x^{3}-90 x^{2}-3696 x-3008\right) y .
\end{aligned}
$$

It is obviously that $n_{1,+}=n_{1,0}=n_{1,-}=0$, and the polynomial $\operatorname{res}_{\mathrm{f}_{1}, \mathrm{f}_{2}}(\mathrm{x})$ may get as following:

$$
\begin{array}{r}
\left(x^{4}+176128-3008 x-1848 x^{2}-30 x^{3}\right) \\
\left(x^{6}-32848 x^{2}-2625780 x-45 x^{5}+\right. \\
\left.24345 x^{3}-249 x^{4}-5223856\right) .
\end{array}
$$

It is not difficult to show that $n_{2,+}=n_{2,-}=2, n_{2,0}=0$.

Thus we have that the inertia is $(2,0,2)$, which the same as the result in [24].

\section{ACKNOWLEDGMENT}

This work was supported by a grant from the National Science Foundation of Ningbo City under grant No.2009A610078, the research foundation of Ningbo University under grant No.xk109042 and No.xk109043. The authors are deeply grateful to the referee for valuable remarks and suggestions.

\section{REFERENCES}

[1] Gantmacher F R, "The theory of matrices", New York: Chelsea, 1959.

[2] Li enard-Chipart, "Sur la signe de partie reelle des raciness d'une equation algebrique", J. Math. Pures Appl., 1914, 10(6), pp. 291-346.

[3] Olga M. Katkova, Anna M. Vishnyakova, "A sufficient condition for a polynomial to be stable", J. Math. Anal. Appl. 2008, (347), pp. 81-89.

[4] Xiaojing Yang, "Some necessary conditions for Hurwitz stability", Automatica, 2004, (40), pp. 527-529.

[5] Xiaojing Yang, "Necessary conditions of Hurwitz polynomials", Linear Algebra and its Applications, 2003, (359), pp. 21-27.

[6] Xiaojing Yang, "Generalized form of Hurwitz-Routh Criterion and hopf bifurcation of high order", Applied M athematics Letters, 2002 (15), pp. 615-621.

[7] Alberto Borobia, Sebastian Dormido, "Three coefficients of a polynomial can determine its instability", Linear Algebra and its Applications, 2001, (338), pp. 67-76.

[8] G. Heining and K. Rost, "Using Algebraic Geometry", Springer-Verlag, New York, 1989.

[9] Waerden Vander B L, "Algebra", Springer-Verlag, New York, 1956.

[10] Yang Lu, "Recent Advances on Determining the Number of Real Roots of Parametric Polynomial", J ournal of Symbolic Computation, 1999, (28), pp. 225- 242.

[11] Yang Lu, Hou Xiaorong, Zeng Zhenbing, "A Complete Discrimination System for Polynomials", Science in China (series E), 1996, 39(6), pp. 628-646.

[12] Yang Lu, Xia Bican, "An Explicit Criterion to Determine the Number of Roots in An Interval of A Polynomial", Progress in Natural Science, 2000, 10(12), pp. 897-910.

[13] K.Ogata, "Discrete-time Control System" $2^{\text {nd }}$ ed., Englewood Cliffs, NJ: Prentice-Hall, 1995.

[14] E. I. Jury, "Theory and application of the z-transform method", New York NY: Wiley, 1964.

[15] Stephen Barnet, Polynomials and Linear Control Systems, New York, Marcel Decker, Inc., 1983.

[16] Xie Liejun, "A Note on Sturm Theorem", Journal of $M$ athematics in Practice and Theory, 2007, 37(1), pp. 121125(in Chinese).

[17] Luca Gemignani, "A fast iterative method for determining the stability of a polynomial", J ournal of Computational and Applied Mathematics, 1996, (76), pp. 1-11.

[18] Luca Gemignani, "Computationally efficient applications of the Euclidean algorithm to zero location", Linear Algebra and its Applications, 1996, (249), pp. 79-91.

[19] G. Heining and K. Rost, "Algebraic Methods for Toeplitzlike Matrices and Operators", Birkhauser, Boston, 1984.

[20] M. G. Krein and M. A. Naimark, "The method of symmetric and Hermitian forms in the theory of separation of the roots of algebraic equations", Linear and Multilinear Algebra, 1981, (10), pp. 265-308.

[21] Luca Gemignani, "Compute the inertia of Bezout and Hankel matrix", CALCOLO, 1991, (28), pp. 267-274.

[22] I S IOHVIDOV. "Hankel and Toeplitz Matrices and Forms", Birkh\$lddot\{a\}\$user, Boston, 1984.

[23] Feng Qin Rong, "A fast method to compute the inertia of Bezout matrix and its application", Chinese Quarterly J ournal of Mathematics, 2001, (16)1, pp. 52-58.

[24] Luca Gemignani, "A hybrid approach to computation of the inertia of a parametric family of Bezoutians with application to some stability problems for bivariate polynomials", Linear Algebra and its Applications, 1998, (283), pp. 221-238. 
Liejun Xie was born in Zhejiang Province of China on April 24, 1974. He received the M.S. degree in Fundamental Mathematics. His main research interests include the mechanization for mathematics, Rough set and its application and mathematical education research.

Currently, he works in the Mathematics Department of Ningbo University. $\mathrm{He}$ is an associate professor. $\mathrm{He}$ has published 17 research papers in journals and international conferences in mathematical and computer field. Some papers of them have been indexed by MR, EI and ISTP: Two theorems for determining $\mathrm{p}$-irreducibility of binding polynomials with degree four(China, Pure Appl. Math. 24, No. 2, 314-320,2008); Some results for determining p-irreducibility of binding polynomials (China, J. Zhejiang Univ. Sci. Ed. 35, No.3, 241247, 2008); A note on the Sturm theorem(China, Math. Practice Theory, 37, No.1 121-125, 2007); A new algorithm for determining sign of real algebraic numbers (China, Bull. Of Sci. and Tech. 23, No.3, 303-307, 2007). He has presided or participated 12 research projects including National Research Projects, Provincial and City Research Projects.

Mr. XIE is the associate deputy of the Department of Mathematics at Ningbo University, the deputy of Mathematical laboratory. He covered himself with honorary of excellent theses in science field of Zhejiang province in 2005. 